\title{
FORMAČ̃̃O DE GESTORES EDUCACIONAIS E ESCOLARES NO CONTEXTO DAS TENDÊNCIAS DAS REFORMAS EDUCACIONAIS: CONSENSOS E DISSENSOS.
}

\author{
Ana Paula Oliveira RESCIA ${ }^{1}$ \\ João Augusto GENTILINI ${ }^{2}$
}

Resumo: Este trabalho apresenta partes dos resultados de uma pesquisa de doutoramento sobre a formação dos gestores educacionais e escolares para o início do século XXI no contexto da descentralização e das tendências das reformas educacionais na América Latina ocorridas no final da década de 1980 e nos anos de 1990. Norteados por uma pesquisa qualitativa, de natureza bibliográfico-documental, os procedimentos metodológicos tiveram referência em estudos realizados por organismos internacionais como a UNESCO, a CEPAL e o ILPE, além de estudiosos dos diferentes paradigmas de gestão, considerando as necessidades dos sistemas de ensino latino-americanos. No caso do Brasil, buscou-se compreender as exigências de transformação de competências locais em planejamento e gestão da educação à luz de implicações e iniciativas de formação de gestores dentro desta nova realidade. Para tanto, analisou-se, com propósitos comparativos, três programas de formação de gestores educacionais e escolares em atuação na rede pública de ensino: Programa Circuito Gestão; Programa de Capacitação a Distância para Gestores Escolares (Progestão) e o Programa Escola de Gestores da Educação Básica Pública. Pretendeu-se identificar as tendências de cada programa em relação à formação dos gestores educacionais e escolares, tendo em vista as transformações ocorridas na sociedade e na educação e as exigências de aquisição de novas competências e habilidades. As conclusões da pesquisa indicaram que apesar de os programas de formação terem se originado em instâncias diferentes e explicitarem, em seus pressupostos, orientações diversas, convergem para o mesmo conjunto de competências na formação de gestores educacionais e escolares.

Palavras-chave: Reformas educacionais. Formação de gestores educacionais e escolares. Competências e habilidades.

\section{INTRODUÇÃO}

A formação de gestores da educação na América Latina é uma necessidade reconhecida pelos organismos internacionais como o Instituto Internacional de Planejamento Educacional (ILPE), conforme mencionado pelo próprio instituto (ILPE,

\footnotetext{
1 Departamento de Educação. UNESP - Universidade Estadual Paulista "Júlio de Mesquita Filho". Faculdade de Ciências e Tecnologia. Presidente Prudente - SP - Brasil. 19060-900 anapaularescia@fct.unesp.br.

${ }^{2}$ Programa de Pós-Graduação em Educação Escolar. UNESP - Universidade Estadual Paulista "Júlio de Mesquita Filho". Faculdade de Ciências e Letras de Araraquara. Araraquara - SP - Brasil. 14800-901 jagentilini@bol.com.br.
} 
2000) a Comissão Econômica para a América Latina e o Caribe (CEPAL) e a Organização das Nações Unidas para a educação, a ciência e a cultura (UNESCO) e pelos órgãos oficiais de gestão da educação pública em vários países latino-americanos que realizaram reformas educacionais de natureza descentralizadora a partir dos anos de 1990. Tem-se propugnado pela necessidade de profissionalização dos gestores em função do papel estratégico que a educação passou a ter frente às transformações produtivas, tecnológicas e nos meios de comunicação da chamada sociedade do conhecimento e da informação.

No caso do Brasil, com a implantação dos sistemas de ensino nos estados e municípios, reconhecidos como entes federativos autônomos, abriu-se uma possibilidade para que a União, Estados e Municípios promovessem iniciativas neste sentido, mas elas têm ocorrido de forma fragmentada e desarticulada considerando-se as três instâncias de gestão da educação (municipal, estadual e federal).

Ao término da primeira década do século XXI e, no caso brasileiro, a quase vinte anos da implementação da atual LDB (RESCIA et al., 2007) e após a aprovação tardia de um novo Plano Nacional de Educação, a gestão da educação nos municípios e nas escolas parece realizar-se, muitas vezes, ainda de forma improvisada e com ações anacrônicas.

Apesar da instituição dos sistemas municipais de ensino (SAVIANI, 1999; ROMÃO, 1997) constituir um fato recente na organização da educação no Brasil e, como tal, pouco compreendido como inovação de gestão e, consequentemente, ainda pouco discutido na pesquisa educacional, a grande maioria dos municípios continua se reportando às normas federais e estaduais de organização do ensino e têm encontrado dificuldades em operacionalizar a organização de seus próprios sistemas, preferindo compartilhar com o Estado, a responsabilidade de administrar a educação no âmbito de seus territórios, adiando para um futuro incerto a possibilidade de dar um salto qualitativo em relação ao desenvolvimento da educação municipal, por meio de formas inovadoras quanto as suas realidades específicas no que diz respeito à gestão da educação local.

É importante esclarecer que o estabelecimento dos sistemas municipais de ensino foi precedido pelo processo de municipalização, iniciado na década de 1990 simultaneamente à reforma do Estado no Brasil e acabou sendo confundido com esse processo quando, na realidade, era uma consequência de um dos princípios de reorganização da educação no Brasil - a descentralização educacional, ao lado da 
autonomia e da gestão democrática. A municipalização das escolas não retirou seu caráter de rede escolar (somatório de escolas submetidas às políticas e às normas da educação na localidade), mas o caráter sistêmico que pretendia, tendo um projeto político-pedagógico local como suporte, acabou sendo prejudicado (GENTILINI, 2010).

Talvez, uma das alternativas para que a construção dos sistemas municipais de educação e sua potencialização de forma autônoma (respeitando-se as diretrizes nacionais de educação, especialmente a gestão democrática em todos os níveis) esteja muito mais na dependência de iniciativas inovadoras dos gestores da educação locais, do que da ação dos que exercem funções de gestão nas esferas centrais e intermediárias do sistema educacional e que, historicamente, têm tutelado os municípios para garantir a implementação de políticas elaboradas com escassa participação das localidades e de suas escolas.

Portanto, se a intenção das novas diretrizes educacionais no Brasil, como está na LDB é o alcance de um exitoso sistema público de educação básica no âmbito de um sistema nacional de educação, os sistemas municipais de ensino podem dar uma contribuição decisiva, a partir de uma gestão mais democrática na organização de suas unidades escolares e da educação municipal (BRASIL, 1996, 1988). Neste sentido, o papel a ser exercido pelos gestores da educação nas localidades e, sobretudo, nas escolas é de suma importância, desde que tenham a formação necessária para assumir essa tarefa.

Considerando esta problemática, que está longe de ser esgotada, apresentaremos o recorte dos resultados de uma pesquisa de doutoramento (RESCIA, 2011), mas limitando-a a uma das dimensões importantes do planejamento e da gestão da educação: as novas exigências colocadas para os gestores da educação nas localidades ante as novas responsabilidades do sistema de ensino, a instituição de capacidades institucionais nas localidades e a formação necessária colocada para tirar proveito da descentralização, em favor do desenvolvimento das escolas e dos municípios.

Dessa forma, as questões fundamentais que permearam nosso estudo foram: Há iniciativas de formação de gestores da educação em desenvolvimento atualmente em municípios do Estado de São Paulo? Estas iniciativas atendem às necessidades de organização e, consequentemente, de gestão e de desenvolvimento dos sistemas de ensino e de suas escolas? O modelo paradigmático tradicional de gestão da educação 
ainda subsiste nos cursos de formação continuada de gestores da educação a quase duas décadas da implementação da LDB?

\section{Descrição do trabalho desenvolvido}

Este trabalho com recorte originado na tese de doutoramento de Rescia (2011) apresenta partes de uma pesquisa qualitativa, de natureza bibliográfico-documental, em que os procedimentos metodológicos tiveram referência em estudos e discussões realizadas por instituições internacionais como a UNESCO, a CEPAL e o IIPE e também em estudiosos dos diferentes paradigmas de gestão educacional e escolar, tendo em vista as necessidades dos sistemas de ensino latino-americanos para o século XXI. Entre eles, destacamos autores que discutem a gestão e o planejamento educacional em função do contexto político latino-americano pós-crise dos anos 1980, como Mattos (1988), Brunner e Sunkel (1993), Cosse (1999) e, mais recentemente, já configurando um novo paradigma de gestão, autores como Sander (1995), Nogueira (1995), Kliksberg (2001), Tedesco (1998) e Casassus (2002), entre outros.

Como forma de discutir e de estudar os diferentes paradigmas utilizados na formação dos gestores educacionais e identificarmos os parâmetros nos quais os municípios poderiam se basear para a promoção (isolada ou em parcerias com as demais instâncias federativas) de cursos de formação de gestores e, consequentemente, aqueles que atendiam com êxito as questões de formação de gestores educacionais e escolares, efetuamos inicialmente, uma discussão sobre o contexto histórico que motivou a busca destes novos paradigmas para a formação do que seria a gestão e os gestores educacionais para o início do século XXI.

Para a compreensão do contexto histórico foram utilizados alguns dos estudos (ILPE, 2000; MATTOS, 1988; COSSE, 1999) que buscam analisar as características mais gerais e comuns aos países latino-americanos referentes aos processos de centralização e descentralização da educação, a emergência dos municípios e localidades e, no caso do Brasil, a implantação dos sistemas de ensino, e as exigências de formação de competências locais em planejamento e gestão da educação - o que implicaria em iniciativas de formação de gestores dentro desta nova realidade.

Os elementos obtidos no primeiro passo da pesquisa possibilitaram a construção de uma perspectiva sobre os pressupostos de formação dos gestores educacionais quanto às competências e habilidades exigidas, tendo em vista as necessidades educacionais da 
educação para o início do século XXI, a gestão democrática dos sistemas de ensino e na busca de qualidade.

Esta perspectiva apontou para um segundo passo, ou seja, a necessidade de verificar a existência de iniciativas (cursos, seminários etc.) de formação de gestores em que aqueles pressupostos de formação estivessem (ou não) presentes, e em que medida os municípios estavam efetivamente utilizando sua autonomia, em face aos processos de descentralização, para tomar iniciativas neste sentido, dentro de suas necessidades e realidades.

Dados empíricos acerca de iniciativas de formação de gestores educacionais desde o início dos anos 2000 no Brasil, nos fez analisar a preocupação dos órgãos dirigentes de dotar os municípios e escolas de condições para implementar as políticas educacionais construídas na década de 1990 e sob orientação da atual LDB; e a partir do contexto de reforma do Estado e dos novos parâmetros de gestão pública, permitiu-nos fazer um recorte no universo da pesquisa que, desta forma, tomou como referência alguns programas de formação de gestores municipais e escolares, realizados em municípios-polo e que reuniram, inicialmente, gestores escolares, através de parcerias entre o governo federal, a universidade pública e os municípios que já haviam instituído seus sistemas municipais de ensino, a saber: Programa Circuito Gestão; Programa de Capacitação a Distância para Gestores Escolares (Progestão) e o Programa Escola de Gestores da Educação Básica Pública.

Como terceiro e último passo, comparamos os programas de formação mencionados, a partir de categorias extraídas dos pressupostos teóricos sobre a formação do novo gestor educacional e escolar para o início do século XXI, ou seja, "competências gerenciais", "competências político-pedagógicas" e "competências para a ação social" e, também, sobre a estrutura organizacional dos referidos programas, tendo como referência a capacidade de criar nos gestores em formação a capacidade (ou a competência) institucional de interferir na orientação das referidas iniciativas, a partir da existência (ou não) de um projeto político educativo e poder local autônomo e no âmbito do sistema municipal de ensino.

\section{Resultados obtidos}

Inicialmente, é importante mencionar que os três programas de formação investigados foram propostos e realizados em um contexto de descentralização do sistema educacional brasileiro, tendo a gestão democrática da educação como diretriz 
nacional. Esperar-se-ia que fossem programas flexíveis e voltados para os gestores municipais e escolares das regiões e localidades, considerando-se os seus interesses peculiaridades. Também que houvesse uma articulação entre as três instâncias de poder (federal, estadual e municipal) no sentido de que frente às peculiaridades regionais e locais, não se perdesse de vista a articulação sistêmica da gestão da educação nacional. A participação das universidades nos programas de formação também foi uma recomendação insistentemente colocada naquele momento, considerando-as não apenas como uma instância de pesquisa e produção de conhecimentos no campo da gestão que só teriam sentido se fossem colocados à disposição da sociedade, mas também como espaço formativo de futuros gestores e dirigentes de educação dentro dos novos paradigmas.

Mas pode-se constatar que os formatos organizacionais de cada programa se diferenciaram. O Programa Circuito Gestão, de iniciativa do Governo do Estado de São Paulo, foi um programa de vasta mobilização, recursos gigantescos, convênios e parcerias com as Prefeituras Municipais e também com a iniciativa privada (restaurantes, hotéis, empresas de transportes etc.), mobilizando mais de vinte mil gestores (aqui compreendidos como todos os que atuam na equipe de gestão das escolas, como diretores, vice-diretores, coordenadores pedagógicos, supervisores de ensino). Valeu-se de consultorias públicas, no caso, de consultores de universidades e também de consultorias privadas. Não houve a participação do governo federal. O Progestão, também iniciativa do Governo do Estado de São Paulo, não teve a mesma dimensão, sendo mais regionalizado e ficou aos cuidados das diretorias de ensino (na época, regionais e municipais).

O Programa Escola de Gestores pareceu-nos como o mais adequado em termos do que se esperava de programas de formação de acordo com as recomendações da UNESCO (2002), ou seja, que fossem localizados e regionalizados, sem grandes mobilizações e com parceria entre governo federal, universidades públicas e secretarias municipais de educação. Assim, pareceu-nos, que apenas esse programa estava mais próximo do parâmetro de formação continuada discutido nos anos de 1990, quando havia uma preocupação com relação aos resultados da descentralização no que se referia à melhoria da qualidade do ensino na América Latina, na medida em que a descentralização exigiria uma formação de gestores em todos os níveis (órgãos centrais, regionais e locais de ensino e nas próprias escolas), para se criar competências 
específicas diante do novo contexto educacional, começando com uma redefinição de função e de gestores públicos.

Ao analisar as supostas competências pretendidas pelos programas de formação pesquisados, notamos que as chamadas competências e habilidades, muitas vezes, se caracterizavam e se confundiam na realidade com objetivos dos próprios programas, além de compreendermos ainda tais competências como sendo resultados de aprendizagens ou atribuições legais que competem aos gestores educacionais e escolares no exercício de seus cargos e/ou funções.

Notamos que o extenso rol de competências, habilidades, capacidades etc., que desde os anos de 1990, vem se acumulando nos programas de formação continuada de gestores educacionais e escolares na América Latina, reflete em consensos e dissensos tanto em seus formatos organizacionais quanto em seus objetivos e metodologias de trabalho. Haveria, entretanto, um modelo de competências e habilidades que fosse predominante? Tendo este foco, julgamos necessário estabelecer alguns critérios para realizar a análise comparativa dos três programas realizados no Estado de São Paulo - a propósito, o estado que mais realizou programas de formação e de qualificação de gestores em relação aos demais estados da federação, desde os anos de 1990, em um contexto de descentralização da educação.

Ao apresentamos uma classificação horizontal de competências e habilidades que optamos por classificar, para efeito didático, como "competências gerenciais", "competências político-pedagógicas" e "competências ético-sociais", identificamos o que seriam possíveis competências e habilidades apontadas em cada um dos três programas. Constatamos que muitas delas não se enquadravam, rigorosamente falando, nos conceitos sobre competências e habilidades, sendo muito mais objetivos desses programas, atribuições legais ou simplesmente, resultados de aprendizagem.

No entanto, é importante esclarecer que entendemos como atribuições legais, o que está definido na legislação normativa do ensino e nas diretrizes de organização dos sistemas educacionais, como obrigações específicas do cargo ou da função do gestor. Todavia, é nosso entendimento, que estas atribuições, podem em determinadas circunstâncias, gerar a necessidade de se desenvolver competências e habilidades dos gestores, apontando para o que é objetivo consensual dos programas de formação, ou seja, a profissionalização.

Ao analisarmos as matrizes teórico-metodológicas dos programas constatamos que suas propostas estavam em disputa no sentido de procurar criar um modelo de 
competências distinto, aparentemente, ao que estava posto. Todavia, os programas, na realidade, convergiram para o mesmo lugar e, assim, uma perversidade (DAGNINO, 2004) estaria colocada no fato de que, apontando para direções opostas e até em muitos momentos de maneira antagônica (dissensos) os três programas convergiram para o mesmo fim (consensos), ou seja, esperava-se a formação de um gestor com competências amplas e complexas capaz de dirimir, ou ao menos minimizar, as mazelas educacionais e escolares, no que diz respeito ao seu espaço de atuação.

Portanto, havia de fato, uma expectativa dos referidos programas de que o rol de competências proposto fosse adquirido e desenvolvido apenas pelo gestor educacional e escolar, individualmente considerado e, neste sentido, pareceu-nos outra dimensão do que chamamos perversidade, ou seja, a busca de um "super" gestor, capaz de enfrentar todos os problemas advindos da gestão da educação (no município e nas escolas) em contextos de grande complexidade e que ao se depositar neles tamanha carga de responsabilidade e de aquisição de capacidades, acabaria por levá-los a certa apatia ou, pelo menos, dificuldade em romper, na prática, com o paradigma tradicional e burocrático de gestão, e de implantar o paradigma voltado para resultados em termos de qualidade de ensino e de gestão democrática.

Portanto, na realidade, o rol de competências proposto pelos organismos internacionais deveria ser pensado não apenas ao alcance do gestor educacional e escolar - mesmo porque isso seria utópico, mas também, no desenvolvimento de competências da equipe gestora, numa perspectiva integradora e democrática de se entender a gestão da educação, onde cada um (diretor ou gestor, vice-diretor, coordenador pedagógico, supervisor de ensino etc.) com suas competências específicas atenderiam no todo (ou em partes) ao conjunto de competências proposto, tornando possível enfrentar situações complexas no campo da educação e que envolvam a escola, a comunidade, o município, o Estado, enfim, toda a sociedade ou as questões advindas do sistema a que as escolas pertencem (federal, estadual, municipal) e, em última análise, ao sistema nacional de educação.

\section{Considerações finais}

Considerando partes dos resultados da pesquisa realizada que deu origem a este trabalho entendemos que as poucas e isoladas iniciativas de formação atual oferecida aos gestores da educação ainda não atendem, efetivamente, às necessidades de organização e, consequentemente, de gestão, de planejamento e de desenvolvimento dos 
sistemas municipais de educação e de suas unidades escolares, e que o modelo paradigmático tradicional de gestão ainda subsiste, principalmente devido aos entraves políticos, burocráticos e orçamentários que têm dificultado o alcance da efetiva autonomia dos entes federativos, sobretudo, os municípios na gestão de seus sistemas de ensino, dentro de suas realidades e necessidades de Educação. Acreditamos, entretanto, que é um grande avanço no Brasil algumas iniciativas governamentais (federal, estadual) de implantar propostas de formação de gestores, tendo em vista os esforços para se melhorar os indicadores educacionais brasileiros a partir dos municípios e suas escolas.

Notamos com a realização da pesquisa que a organização e implementação dos programas em análise respondem a uma necessidade, apontada nas recomendações dos anos de 1990 pelos organismos como a UNESCO, a CEPAL e o ILPE para a qualificação e a profissionalização dos gestores em todos os níveis. Entretanto, os programas não responderam a uma exigência sistêmica recomendada para os países que, como o Brasil, promoveu processos de descentralização da educação, ou seja, que todas as instâncias de gestão do sistema nacional de educação se articulem para dar o suporte técnico, operacional, enfim, político-pedagógico aos referidos programas, a partir das diretrizes nacionais de educação, mas observando, também, as peculiaridades e necessidades regionais e locais.

Na medida em que efetuávamos a análise comparativa dos referidos programas identificamos dissensos e consensos nas orientações gerais, objetivos e definição do rol de competências e habilidades desejadas por cada um dos programas, mas foi possível identificar também, certa convergência nos objetivos que chamamos de "perversa" na medida em que, mesmo sendo programas originados de instâncias diferentes do Estado, com orientações político-ideológicas, em tese, divergentes, a formação desejada dos gestores era, se não a mesma, pelo menos bastante similar, ou seja, as características do modelo de competências e habilidades não se afastava muito do modelo recomendado nos anos de 1990, para a formação de gestores em um cenário de transformações tecnológicas, transição e crise política e econômica.

Neste sentido, podemos evidenciar também uma perversidade quando temos o sistema educacional do país, nitidamente, desarticulado do que se espera que seja uma de suas questões mais emergentes, a profissionalização de seus atores e a articulação entre os sistemas. 
Concluímos, portanto, que as poucas e isoladas iniciativas de formação atual oferecida aos gestores educacionais e escolares ainda não atendem, satisfatoriamente, às necessidades de organização e, consequentemente, de gestão, de planejamento e de desenvolvimento dos sistemas municipais de educação e de suas unidades escolares, e que o modelo paradigmático tradicional de gestão ainda subsiste, principalmente devido aos entraves políticos, burocráticos e orçamentários que têm dificultado o alcance da efetiva autonomia dos entes federativos, sobretudo, os municípios, na gestão de seus sistemas de ensino, dentro de suas realidades e necessidades de Educação.

Concluímos, igualmente, que uma das oportunidades para superar esta situação, a partir de programas de formação e profissionalização, estaria no momento da discussão e da implementação dos planos municipais de educação, com o funcionamento adequado da atual precária atuação dos Conselhos Municipais de Educação, dos Conselhos Gestores e dos Conselhos Escolares - de suma importância para o funcionamento democrático dos sistemas municipais de ensino, esclarecendo, conscientizando e proporcionando o suporte teórico-prático para seus integrantes (a começar pela equipe do órgão municipal de ensino) dentro dos novos paradigmas de gestão/planejamento da educação em contextos descentralizados. 
Abstract: This paper shows some of the results from a doctoral research on the educational and school managers training to the beginning of XXI century, in the context of decentralization and trends of educational reforms in Latin America at the end of 1980s and 1990s. Guided by a qualitative, bibliographic and documentary research, the methodological procedures had reference in studies conducted by international organizations, such as: UNESCO, ECLAC and ILPE as well as scholars from different management paradigms, considering the Latin-American education systems' needs. In Brazil, we sought to understand the requirements of transformation of local competences in planning and educational management after implications and managers training initiatives within this new reality. Therefore, it was analyzed for comparative purposes, three educational and school managers training programs in public schools: Management Circuit Program; Distance Learning Program for School Managers and the Managers' School Program of Public Basic Education. It was intended to identify the trends of each program for educational and school managers training, with a view to the changes occurred in our society and education and the requirements to acquire new skills and abilities. As conclusion, the research indicated that although the training programs have originated from different instances and explain various guidelines, everything converges to the same set of skills in educational and school managers training.

Key words: Educational reform. Educational and school managers training. Skills and abilities.

\section{REFERÊNCIAS}

BRASIL. Constituição (1988). Constituição da República Federativa do Brasil. Brasília: MEC, 1988.

Lei Federal $N^{\circ}$ 9.394, de 20 de dezembro de 1996. Dispõe sobre as Diretrizes e Bases da Educação Nacional. Brasília: MEC, 1996.

BRUNNER, J. J.; SUNKEL, G. Conocimiento, sociedad y politica. Santiago de Chile. FLACSO, 1993.

CASASSUS, J. Problemas de la gestión educativa en América Latina: la tensión entre los paradigmas de tipo a y el tipo b. Em Aberto. Brasilia, v.19, n.75, p.49-69, jul. 2002.

COSSE, G. Las lógicas organizacionales en las reformas educativas

latinoamericanas: conflictos y tensiones. Programa para a Reforma Educacional da América Latina (PREAL). Buenos Aires: Diálogo Interamericano, 1999.

DAGNINO, E. Sociedade civil, participação e cidadania: de que estamos falando? In: MATO, D. (Coord.) Políticas de cidadania y sociedad civil em tiempos de globalización. Caracas: FACES, Universidad Central de Venezuela, 2004. p.95-110.

GENTILINI, J. A. Planejamento da educação, Projeto político e autonomia: desafios para o poder local. São Paulo: Cultura Acadêmica, 2010. (Pedagogia, 6). 
ILPE. Formação de recursos humanos para a gestão educativa: informe do fórum realizado no ILPE Buenos Aires, 11-12 novembro 1998. Tradução de C. L. Casta Genovez. Brasília: UNESCO, 2000. (Cadernos UNESCO. Série Educação; 4

KLIKLSBERG, B. O desafio da exclusão: para uma gestão social eficiente. São Paulo: Editora da FUNDAP, 2001.

MATTOS, C. A. de. La descentralización: uma nueva panacéia para impulsar el desarrollo local? Socialismo y Participación, Madrid, n.46, p.23-42, 1988.

NOGUEIRA, R. R. La transformación del modelo de organización y gestión educativa: el aprendizaje acumulado. In: Innovaciones em la gestión educativa. Santiago de Chile: OREALC/UNESCO, 1995. p.11-39.

RESCIA, A. P. O. Consensos e dissensos na formação de gestores educacionais e escolares no Estado de São Paulo. 2011. 144f. Tese (Doutor em Educação Escolar) Faculdade de Ciências e Letras de Araraquara, Universidade Estadual Paulista, Araraquara, 2011.

et al. (Org.). Dez anos de LDB: contribuições para a discussão das políticas públicas em educação no Brasil. Araraquara: Junqueira\&Marin, 2007.

ROMÃO, J. E. Política de ensino básico nas municipalidades. In: CONGRESSO DE ARTES E CIÊNCIAS HUMANAS, LETRAS E ARTES, 3, 1997, Juiz de Fora. Anais... Juiz de Fora: UFJF, 1997. Mimeografado.

SANDER, B. Gestão da educação na América Latina: a construção e a reconstrução do conhecimento. Campinas: Autores Associados, 1995.

SAVIANI, D. Sistemas de ensino e planos de educação: o âmbito dos municípios. Educação \& Sociedade, Campinas, vol.20, n.69, dez. 1999.

TEDESCO, J. C. O novo pacto educativo: educação, competitividade e cidadania na sociedade moderna. São Paulo: Ática, 1998.

UNESCO. Educação na América Latina: análise de perspectivas. Brasília: UNESCO, OREALC, 2002. 